\title{
Using scent detection dogs to identify environmental reservoirs of Clostridium difficile: Lessons from the field
}

\author{
Cheng Li, BSc; ${ }^{1}$ Teresa Zurberg, RCT; ${ }^{3}$ Jaime Kinna; ${ }^{3}$ Kushal Acharya, BSc; Jack Warren, BSc; ${ }^{3}$ Salomeh Shajari, BSc; ${ }^{3}$ \\ Leslie Forrester, BA, MA, MSc; ${ }^{3}$ Elizabeth Bryce, MD, FRCPC ${ }^{1,2}$ \\ ${ }^{1}$ Faculty of Medicine, University of British Columbia, Vancouver, BC, Canada \\ ${ }^{2}$ Division of Medical Microbiology and Infection Control, Vancouver Coastal Health, Vancouver, BC, Canada \\ ${ }^{3}$ Patient Quality and Safety, Vancouver Coastal Health, Vancouver, BC, Canada
}

Corresponding author:

Elizabeth Bryce, MD, FRCPC, Vancouver Coastal Health, Vancouver, BC, Canada

elizabeth.bryce@vch.ca

\begin{abstract}
Environmental reservoirs have been implicated in transmission of Clostridium difficile infections. Scent detection by canines has demonstrated promising ability to rapidly triage hospital surfaces and equipment. 18 months of data collected post-implementation of the canine scent detection project at Vancouver Coastal Health were used to identify key environmental reservoirs for $\mathrm{C}$. difficile and possible mitigation strategies.
\end{abstract}

\section{KEYWORDS}

Canine; Clostridium difficile; environmental reservoir; scent detection

\section{INTRODUCTION}

Clostridioides difficile (CD) remains one of the most common causes of nosocomial infections with significant morbidity and cost [1]. In addition to direct physical transmission via contact with colonized surfaces, environmental reservoirs have been implicated and the ability to rapidly triage surfaces for this organism could greatly enhance infection prevention efforts $[2,3]$. Building upon a proof of concept article that used a beagle to detect CD in patients, a Springer Spaniel was trained to detect $\mathrm{CD}$ odour on equipment and environmental surfaces rather than on patients [4]. Previous evaluation of the dog revealed an overall sensitivity of $92.3 \%$ and specificity of $95.4 \%$ for both odour recognition and search capability using gauze exposed to CD odour, and a canine scent detection program was established at the Vancouver General Hospital (VGH) [5]. The objective of this article is to describe the operational aspects of the canine scent detection program and present the findings and lessons learned from 18 months (May 1, 2017 to October 31, 2018) of environmental detection in a tertiary care facility.

\section{METHODS}

Qualification and training of the certified handler and dog The canine scent detection program based at VGH in Canada currently consists of two canine/handler teams. The second validated canine team joined the program in December 2017 (13 months after the first team was introduced) and consists of a four-year-old Springer Spaniel and a handler with over ten years of experience raising, training, and handling narcotic and explosive detection dogs. The handler had previously been validated through the Justice Institute of British Columbia's security dog program and holds a diploma in Canine Behavior Science and Technology through the Companion Animal Sciences Institute. Additional information on the original canine team and the annual validation process are detailed in a previous paper [5].

\section{The scent detection program}

The team searches every clinical unit and area in the hospital on a monthly basis but also focuses on areas of higher risk, as follows: a) clinical areas with the highest rates of $\mathrm{CD}$; b) any unit with new $\mathrm{CD}$ cases; $\mathrm{c}$ ) any unit with a previous history of high number of canine alerts; and d) units that have had recent cluster events with antibiotic-resistant organisms (e.g., Methicillin-resistant Staphylococcus aureus).

Each day, before the canine teams begin their searches, a quality control assessment is performed using scent pads from known positive $\mathrm{CD}$ fecal samples and from $\mathrm{CD}$ cultures. These are hidden by a third party and the team is evaluated for its ability to find the sample. The canine team then proceeds to the identified units/clinical areas for that day. Details of each search and alert are entered into an Access database for analysis and report generation. Alerts by the canine team have been categorized into a) general environmental; b) patient

Acknowledgements: We appreciate the invaluable contributions to this project provided by the ward nurses and staff and the infection control team at Vancouver General Hospital. We also recognize the funding support provide by the VGH \& UBC Hospital Foundation.

Conflicts of interest: None.

Funding: None. 
room; and c) washroom alerts. General environmental alerts include areas accessible to the general public, patients, and healthcare workers (HCWs) (e.g., hallway, waiting rooms), while patient room alerts are those occurring in unoccupied rooms (including en-suite washrooms). The canine team does not search occupied patient rooms. Washroom alerts include shared patient, public, and staff washrooms.

\section{RESULTS}

Between May 1, 2017 and October 31, 2018, 659 clinical areas at VGH were searched over 115 search days (5.7 clinical areas/day). Each area took approximately 30 minutes to be searched, depending on the complexity of the area and the number of positive alerts - this did not include report generation, notification, and actionable events. During this time, there were 391 alerts on items for an average of 0.6 alerts per clinical area. Statistical results of these clinical sweeps, as well as subcategories of alerted items based on various environments and locations, are detailed in Table 1. Table 2 details the results of the general, patient room, and washroom alerts. A total of $82.1 \%(321 / 391)$ of all positive alerts occurred in the general environment, with 192/321 (59.8\%) alerts on items (e.g. carts, DINAMAP ${ }^{\mathrm{TM}}$, staff lockers) that were almost exclusively handled by HCWs.

\section{DISCUSSION}

The canine team alerts confronted our presumptions of where $\mathrm{CD}$ reservoirs lie and challenged us to re-examine the way we approach infection prevention. The results highlight the impact of cross-transmission not only by HCWs, but also by patients and the public, as evidenced by the alerts in public washrooms and both patient/family as well as staff lounges and lockers. Hallway items alone accounted for 219/391 (56\%) of all alerts, emphasizing the importance of decluttering to permit effective cleaning. Other alerts highlighted items that could be addressed by re-engineering or a systems change. For example, the insides of toilet paper dispensers were positive (likely from individuals with contaminated hands reaching up for toilet paper). Changing the dispenser design and/or the quality of the toilet paper could address this issue. Alerts on the tube system (used to transport patient specimens) resulted in the purchase of cleanable "landing" mats and a review of the protocols for regular tube cleaning.

\section{TABLE 1: Canine search statistics.}

\begin{tabular}{|l|l|}
\hline Areas Searched and Alerts & Counts (\%) \\
\hline Search days & 115 \\
\hline Areas searched* & 659 \\
\hline Areas with positive alerts & $\begin{array}{l}317(48.1 \% \text { of all areas } \\
\text { searched })\end{array}$ \\
\hline $\begin{array}{l}\text { Number of items with positive } \\
\text { alerts }\end{array}$ & 391 (1.2 positive items/area) \\
\hline General environment alerts & $321(82.1 \%$ of all alerts $)$ \\
\hline Patient room alerts & $40(10.2 \%$ of all alerts $)$ \\
\hline Washroom alerts & $30(7.7 \%$ of all alerts) \\
\hline $\begin{array}{l}\text { *Areas = clinical units and patient support services } \\
\text { (e.g. radiology). }\end{array}$ \\
\hline
\end{tabular}

TABLE 2: Alerts in the general environment, patient room, and shared washroom environments.

\begin{tabular}{|c|c|}
\hline Alert Environment & $\begin{array}{l}\text { Count }(\% \text { of } \\
\text { Total Alerts) }\end{array}$ \\
\hline General environment & $321(82.1 \%)$ \\
\hline Hallway & $219(56.0 \%)$ \\
\hline Clean storage area & $35(9.0 \%)$ \\
\hline Staff lounges/lockers & $33(8.4 \%)$ \\
\hline Patient lounges/common areas & $18(4.6 \%)$ \\
\hline Nursing station & $9(2.3 \%)$ \\
\hline Miscellaneous & $6(1.5 \%)$ \\
\hline \multicolumn{2}{|l|}{ Top ten items alerted on in general environment: } \\
\hline Cart* & $71(18.2 \%)$ \\
\hline DINAMAP ${ }^{\mathrm{TM} * *}$ & $22(5.6 \%)$ \\
\hline Staff locker & $19(4.9 \%)$ \\
\hline Chair & $13(3.3 \%)$ \\
\hline $\begin{array}{l}\text { Bed (frame, handrails, bedding, } \\
\text { pillows)*** }\end{array}$ & $12(3.1 \%)$ \\
\hline Wheelchair & $11(2.8 \%)$ \\
\hline Pillow (not on bed) & $9(2.3 \%)$ \\
\hline Sling & $9(2.3 \%)$ \\
\hline Patient chart & $8(2.0 \%)$ \\
\hline Tube station & $7(1.8 \%)$ \\
\hline Cabinets & $7(1.8 \%)$ \\
\hline Supply bins & $6(1.5 \%)$ \\
\hline Patient environment & $40(10.2 \%)$ \\
\hline Items alerted on in patient bed area & $31(7.9 \%)$ \\
\hline Items alerted on in washroom & $9(2.3 \%)$ \\
\hline \multicolumn{2}{|l|}{ Top two items alerted on in patient room: } \\
\hline Cart & $8(2.0 \%)$ \\
\hline Bed (frame, handrails, bedding, pillows) & $5(1.3 \%)$ \\
\hline Common washroom environment & $30(7.7 \%)$ \\
\hline Shared patient bathrooms & $26(6.6 \%)$ \\
\hline Staff washrooms & $3(0.8 \%)$ \\
\hline Public washrooms & $1(0.3 \%)$ \\
\hline \multicolumn{2}{|l|}{ Top two items alerted on in washrooms: } \\
\hline Toilet paper holder & $10(2.6 \%)$ \\
\hline Commode & $5(1.3 \%)$ \\
\hline \multicolumn{2}{|c|}{$\begin{array}{l}\text { *Includes medication, personal protective equipment, } \\
\text { resuscitation, glucometer, phlebotomy, housekeeping, and } \\
\text { clean linen carts. } \\
* * \text { DINAMAP }{ }^{\mathrm{TM}} \text { is a machine that measures and monitors } \\
\text { various vitals, including blood pressure, temperature, oxygen } \\
\text { saturation, and pulses. } \\
* * * \text { Some beds are located outside the patient environment } \\
\text { (e.g., hallways). }\end{array}$} \\
\hline
\end{tabular}

Of note, the canine teams are not asked to search occupied rooms, including those that are known to house patients with $C D$, and empty rooms not yet cleaned and disinfected. A decision was made early in the program that the information would not be useful in terms of directing environmental cleaning efforts. Further, it could put the canine team at additional, 
unnecessary risk of exposure to $\mathrm{CD}$. The majority of rooms that are searched have undergone terminal cleaning and disinfection and await new patient occupation. Similarly, while the dogs occasionally alert on the floors, they have been taught during training that searching floors and garbage is not of "value" for a reward. This is for both safety and pragmatic reasons: floors are considered dirty from an infection prevention perspective and for obvious reasons, having the dogs search garbage or floors is not practical. The dogs understand relative situational search environments. An example of this is that the dogs know the handler has a hide in their vest but does not continuously alert on that hide until it is placed and the search command is given.

The fact that the dogs are trained on the odour only of CD (rather than on fecal specimens) leads us to believe that the dogs alert on the volatile organic compound signature. This has been indirectly confirmed by the fact that, in clinical practice, the dogs often search the re-cleaned area and rarely alert. This also suggests that ultraviolet $\mathrm{C}$ light and/or hydrogen peroxidebased cleaning/disinfection are adequate for removing volatile organic compounds (and associated organisms) from the environment. The program is still at the formative stages of research into biological scent detection and these are questions that the program hopes to address in the future.

One of the difficulties with achieving compliance with infection prevention measures is the lack of visible cause and effect as well as the delayed presentation of infection, making accountability less visible [6]. A positive canine alert now results in immediate notification of unit staff and hospital environmental services (EVS) for priority cleaning/disinfection of the room or equipment and use of ultraviolet $C$ light disinfection, as appropriate, to the item or space identified. Every positive alert is considered to be an opportunity for "in-the-moment" team discussion and feedback regarding routes of transmission and cleaning/disinfection efforts. Changing the collective norm is a very important aspect of behaviour change and engaging both HCWs and the public with the use of canine teams is a positive way of highlighting and reinforcing ideal behaviour [7]. The canine/handler team provides a visual reminder of the importance of environmental reservoirs in infection transmission and emphasizes the modes of transmission to HCWs in a non-punitive way. While it is difficult to prove that the scent detection program by itself decreases the incidence of $C D$, the highly visible presence of dog handlers and dogs likely improves compliance with infection prevention measures such as hand hygiene, disinfection of personal items, and appropriate use of personal protective equipment.

Limitations of the scent detection program include the potential bias introduced by the prioritizing protocol followed by the dog handlers. Furthermore, the distribution of alerts by item type is influenced by the total number of those items. Lastly, while the comparison of EVS cleaning protocols for different items was out of the scope of this study, the bias introduced by the cleaning personnel and the cleaning protocol itself could have had an impact on the number of positive alerts.
In conclusion, as a quality improvement initiative, the scent detection program studies the multifaceted interactions between the environment and key populations, highlighting the interactions between $\mathrm{HCW}$ s and the system with which they work, including the use of devices, the environment, and the complexities of patient care, in the context of CD transmission and prevention. It also allows us to address some key challenges in infection prevention, such as delayed feedback to HCWs, in a safe, non-punitive environment. The authors hope to shape the canine scent detection initiative into a sustainable quality improvement model from certification to implementation.

\section{REFERENCES}

1. Desai, K., Gupta, S. B., Dubberke, E. R., Prabhu, V. S., Browne, C., \& Mast, T. C. (2016). Epidemiological and economic burden of Clostridium difficile in the United States: Estimates from a modeling approach. BMC Infectious Diseases, 16(303), 1-10. doi: https://doi.org/10.1186/s12879016-1610-3

2. Huang, S. S., Datta, R., \& Platt, R. (2006). Risk of acquiring antibiotic-resistant bacteria from prior room occupants. Archives of Internal Medicine, 166(18), 1945-1951. doi: https://doi.org/10.1001/archinte.166.18.1945

3. Shaughnessy, M. K., Micielli, R. L., DePestel, D. D., Arndt, J., Strachan, C. L., Welch, K. B., \& Chenoweth, C. E. (2011). Evaluation of hospital room assignment and acquisition of Clostridium difficile infection. Infection Control \& Hospital Epidemiology, 32(3), 201-206. doi: https://doi. org/10.1086/658669

4. Bomers, M. K., van Agtmael, M. A., Luik, H., VandenbrouckeGrauls, C. M. J. E., \& Smulders, Y. M. (2014). A detection dog to identify patients with Clostridium difficile infection during a hospital outbreak. Journal of Infection, 69(5), 456-461. doi: https://doi.org/10.1016/j.jinf.2014.05.017

5. Bryce, E., Zurberg, T., Zurberg, M., Shajari, S., \& Roscoe, D. (2017). Identifying environmental reservoirs of Clostridium difficile with a scent detection dog: Preliminary evaluation. Journal of Hospital Infection, 97(2), 140-145. doi: https://doi. org/10.1016/j.jhin.2017.05.023

6. Anderson, J., Gosbee, L. L., Bessesen, M., \& Williams, L. (2010). Using human factors engineering to improve the effectiveness of infection prevention and control. Critical Care Medicine, 38(8 Suppl), S269-S281. doi: https://doi. org/10.1097/CCM.0b013e3181e6a058

7. Singer, S. J., Benzer, J. K., \& Hamdan, S. U. (2015). Improving health care quality and safety: The role of collective learning. Journal of Healthcare Leadership, 7, 91-107. doi: https://doi. org/10.2147/JHL.S70115 